\title{
Retinal ganglion cell/inner plexiform layer thickness in patients with Parkinson's disease
}

\author{
Maja Živković ${ }^{1,2}$, Volkan Dayanir ${ }^{3}$, Jelena Stamenović ${ }^{4}$, Srdjan Ljubisavljevićc ${ }^{1,4}$, Ana Pražićc ${ }^{4}$, Marko Zlatanović \\ Gordana Zlatanovići, ${ }^{1,2}$, Vesna Jakšić ${ }^{5}$, Marija Radenković ${ }^{2}$, Svetlana Jovanovićc \\ ${ }^{1}$ Faculty of Medicine, University of Nis, Niš, Serbia, ${ }^{2}$ Ophthalmology Clinic, Clinical Center Niš, Niš, Serbia, ${ }^{3}$ BATIGOZ Health \\ Group Eye Clinic, Cankaya, Izmir, Turkey, ${ }^{4}$ Neurology Clinic, Clinical Center Niš, Niš, Serbia, ${ }^{5}$ Clinic for Eye Diseases "Prof. Dr Ivan \\ Stanković", Clinical-Hospital Center Zvezdara, Belgrade, Serbia, ${ }^{6}$ Faculty of Medical Sciences, University of Kragujevac, Department \\ of Ophthalmology, Kragujevac, Serbia
}

\begin{abstract}
Introduction: The aim of the paper was to analyze the changes in the macular ganglion cell layer and inner plexiform layer (GCL-IPL) thickness in patients with Parkinson's disease.

Material and methods: The study enrolled 46 patients with established diagnosis of Parkinson's disease and 46 healthy subjects. Both groups were age- and gender-matched. An OCT protocol, namely standardized Ganglion Cell Analysis algorithm was used to measure the thickness of the macular GCL-IPL layer. The average, minimum, and six sectoral (superotemporal, superior, superonasal, inferonasal, inferior, inferotemporal) GCL-IPL thicknesses were measured from the elliptical annulus centered on the fovea.

Results: The mean value of the clinical severity of Parkinson's disease was between 2 and 3, according to the Hoehn and Yahr scale. Statistically significant thinning of the GCL-IPL layer was registered in average and minimum GCLIPL thickness, as well as in the sectoral layer thicknesses in patients with Parkinson's disease in comparison to the controls. There was no correlation between structural changes in the retina and disease duration or severity. A statistically significant difference in thickness between the different stages of the disease was registered only in the inferior sector.

Conclusions: Parkinson's disease is accompanied by thinning of the GCL-IPL complex of macula even in the earliest stages. This may indicate a possible retinal dopaminergic neurodegeneration. There is no correlation between duration or severity of Parkinson's disease with thinning of the GCL-IPL complex.
\end{abstract}

Key words: Parkinson's disease, ganglion cell complex, inner plexiform layer, retina.

\section{Introduction}

Parkinson's disease (PD) is a chronic, progressive neurodegenerative disorder mostly affecting the aging population. The most common type of presentation of the disease is idiopathic, although it may occur secondary to the tumor, injury or infection $[14,17]$. Basically, the disease is a movement disorder caused 
by a decrease in a chemical substance, dopamine, in the part of the brain responsible for voluntary motor control (the basal ganglia) $[11,24]$.

Dopaminergic neurons play key roles in the central nervous system (CNS), mediating basic mechanisms of vision, movement, motivation and mood. Dopamine as a neurotransmitter presents in retinal amacrine cells. Dopamine is released by a unique set of amacrine cells and activates D1 and D2 dopamine receptors distributed throughout the retina $[2,5,6]$. Today's research on the retina focuses a great deal of attention on neurotransmission between the neurons of the retina. Dopaminergic amacrine cells are one of the most sparsely distributed retinal nerve cell types found within the mammalian retina [30]. Amacrine cells are inhibitory neurons, and project their dendritic arbors to the inner plexiform layer (IPL), there interacting with retinal ganglion cells and/or bipolar cells [9].

Amacrine cells operate at the IPL, the second synaptic retinal layer where bipolar cells and retinal ganglion cells form synapses. There are at least 33 different subtypes of amacrine cells based just on their dendrite morphology and stratification. Each type of the amacrine cell releases one or several neurotransmitters where it connects with other cells. They are often classified by the width of their field of connection, the layer(s) of the stratum in the IPL they are in, and by the neurotransmitter type $[9,20]$. There is still much to be discovered about all of the different functions of all of the different amacrine cells.

Amacrine retinal cells located in IPL can be measured with the segmentation of the ganglion cell layer and inner plexiform layer (GCL-IPL) by the latest version of Cirrus OCT [11]. Measurement of the GCL-IPL layer may prove to be of value in providing an objective criterion in diagnosis and/or monitoring progression of PD. There are previous works demonstrating decreased visual acuity, contrast sensitivity and color vision defects among patients with PD [1], early-onset thinning of the inner retinal layers on OCT [3,31] and functional alterations observed in retinal ganglion cells [29] may strengthen authors' rationale in conducting this study and can make the readers comprehend the aims of the study better.

The aim of the paper was to analyze the changes in macular GCL-IPL layer thickness in patients with early stages of PD in order to assess whether loss of dopaminergic neurons, i.e. amacrine cells, in the retina of patients with $\mathrm{PD}$ could be demonstrated as thinning of the respective retinal layers on OCT scans, and whether this thinning is correlated with disease severity and/or duration.

\section{Material and methods}

This was a prospective, nonrandomized, observational study conducted between July 2014 and July 2016 at the Neurology Clinic and Eye Clinic, Clinical Center Niš, University of Niš, Serbia. The study followed the tenets of the Declaration of Helsinki. Informed consent was obtained from each patient after explanation of the nature of the study.

Patients with PD were consecutively enrolled as they presented at the Neurology Clinic. The diagnosis of PD was made according to the UK PD Brain Bank criteria [15]. All patients were evaluated by a neurologist who had no access to ophthalmologic findings. All enrolled patients were in a clinically stable phase using substitution therapies at doses of levodopa of $750 \mathrm{mg}$ to $1000 \mathrm{mg}$ per day, in divided doses. Patients who were under treatment for less than one year (6 months to a year) were included. Disease severity was evaluated according to the Hoehn and Yahr (HY) scale [14] which is widely used to categorize the progression of PD symptoms and quantify the patients according to 5 stages.

Exclusion criteria comprised any ocular disease that may confound the assessment of the retina: $\mathrm{AMD}$, diabetic retinopathy, glaucoma, retinal vessel occlusion, retinal dystrophies, uveitis. Patients with myopia and hypermetropia > 2.0 Dsph were excluded. Also patients with previous intraocular surgery were excluded from the study.

All patients underwent a complete ophthalmic examination as follows: best corrected visual acuity (BCVA), intraocular pressure measurement by applanation tonometry, gonioscopy, slit lamp examination of the anterior segment and fundus examination with a plus 90-diopter lens. The OCT protocol was performed according to the standardized operating procedures included in Cirrus SD-OCT device (model 4000, software version 6.0, Carl Zeiss Meditec, Inc.). The Ganglion Cell Analysis algorithm of the aforementioned device was used to process and measure the thickness of the macular GCL-IPL layer. The average (GCavg), minimum (GCmin), and six sectoral (superotemporal, superior, superonasal, inferonasal, inferior, inferotemporal) GCL-IPL thicknesses were measured from the elliptical annulus centered on the fovea. Demarcation of superior sectors was performed from nasal to temporal, thus the superior 
nasal sector of GCL+IPL was marked as GC1, superior as GC2, superior-temporal as GC3, while demarcation of inferior sectors was performed from temporal to nasal, marking inferior-temporal as GC4, inferior GC5 and inferior-nasal as GC6. Images with a signal power more than seven were used for analysis.

A sex- and age-matched normal control group was recruited from normal healthy population. Both eyes of healthy participants had to meet the following criteria: (1) no history or evidence of retinal and eye pathology, (2) no history of ocular surgery, (3) $\mathrm{IOP}<21 \mathrm{~mm} \mathrm{Hg}$, and (4) had to be free of optic nerve damage and without any systemic and neurological disease.

\section{Statistical analysis}

Statistical analysis was done using the statistical package SPSS 22 for Windows (SPSS Inc., USA). Primary data obtained were analyzed by descriptive statistical methods and methods for testing hypotheses. The following descriptive statistical methods were used: measures of central tendency (mean, median), rate variability (standard deviation and variation interval) and indicators of structure expressed in percentages. Kolmogorov-Smirnov and Shapiro-Wilk tests, skewness value ("skewing") and kurtosis ("taper/flatness") were used to determine the normality of distribution. The $t$ test was used to test the difference of arithmetic mean between the groups. Analysis of variance (ANOVA) was used to test the difference between the stages of the dis- ease. The conclusion was done at the level of a statistical significance of 0.05 . Correlation analysis was performed by measuring the correlation of two variables. Spearman correlation coefficient of ranks was used to determine the direction and strength of the connection stages of PD with thickness of GC1-GC6 as well as GCLavg and GCLmin.

\section{Results}

The study included 46 eyes of 46 patients suffering from PD. Out of the 46 patients, 20 were males and 26 were females. The mean age was $66.00 \pm 9.67$ years. Average value of PD stage was 2.49 . The control group was composed of 46 eyes of 46 patients, of which 21 were males and 25 were females with a mean age of $64.47 \pm 11.33$ years.

We analyzed GCL-IPL average thickness (GCavg), minimum thickness (GCmin) and thickness of all six sectors. There was a statistically significant difference between the mean values in the observed and the control groups with respect to all 6 sectors, GCavg and GCmin (Table I).

There was no statistically significant difference in mean values of GC1, GC2, GC3, GC4, GC6, GCavg and $\mathrm{GCmin}$ between the stages of the disease. Only the mean GC5 value was statistically different (ANOVA, $p=0.041$ ) between different PD stages (stages I and II, $p=0.046$; stages II and III, $p=0.012$ ) (Table II).

Correlation analysis between the stages of disease and the value of GC1, GC2, GC3, GC4, GC5, GC6, GCLavg, GCLmin has shown that there was no sta-

Table I. Clinical characteristics and ganglion cell-inner plexiform layer measurements of Parkinson's disease patients and healthy subjects

\begin{tabular}{|c|c|c|c|}
\hline & $\begin{array}{l}\text { Patients with Parkinson's disease } \\
\qquad(n=46)\end{array}$ & $\begin{array}{l}\text { Control group } \\
\qquad(n=46)\end{array}$ & $p$ \\
\hline Age (mean \pm SD) & $66.00 \pm 9.67$ & $64.47 \pm 11.33$ & $0.081^{a}$ \\
\hline Gender (male/female) & $20 / 26$ & $21 / 25$ & \\
\hline GC1 & $72.45 \pm 14.36$ & $86.34 \pm 2.52$ & $<0.001^{a}$ \\
\hline GC2 & $69.77 \pm 15.64$ & $86.55 \pm 2.60$ & $<0.001^{a}$ \\
\hline GC3 & $71.28 \pm 14.10$ & $86.45 \pm 2.58$ & $<0.001^{a}$ \\
\hline GC4 & $72.05 \pm 14.23$ & $86.12 \pm 3.45$ & $<0.001^{a}$ \\
\hline GC5 & $70.97 \pm 15.38$ & $86.62 \pm 2.60$ & $<0.001^{a}$ \\
\hline GC6 & $72.95 \pm 14.23$ & $86.31 \pm 2.73$ & $<0.001^{a}$ \\
\hline GCavg & $72.44 \pm 13.18$ & $86.01 \pm 3.68$ & $<0.001^{a}$ \\
\hline GCmin & $65.33 \pm 19.01$ & $82.49 \pm 1.78$ & $<0.001^{a}$ \\
\hline
\end{tabular}

a t-test

GC1 - superonasal sector of the ganglion cell-inner plexiform layer, GC2 - superior sector of the ganglion cell-inner plexiform layer, GC3-superotemporal sector of the ganglion cell-inner plexiform layer, GC4 - inferotemporal sector of the ganglion cell-inner plexiform layer, GC5 - inferior sector of the ganglion cell-inner plexiform layer, GC6 - inferonasal sector of the ganglion cell-inner plexiform layer, GCavg-ganglion cell-inner plexiform layer average thickness, GCmin-ganglion cell-inner plexiform layer minimum thickness 
Table II. Thickness of the ganglion cell-inner plexiform layer in different stages of Parkinson's disease

\begin{tabular}{|lcccc|}
\hline & Stage I, $n=14$ & Stage II, $n=17$ & Stage III, $n=15$ & $p$ \\
\hline GC1 & $71.57 \pm 15.45$ & $71.42 \pm 16.11$ & $77.71 \pm 6.02$ & $0.358^{\mathrm{a}}$ \\
\hline GC2 & $69.43 \pm 14.96$ & $68.13 \pm 18.52$ & $74.00 \pm 7.50$ & $0.503^{\mathrm{a}}$ \\
\hline GC3 & $70.30 \pm 14.04$ & $70.95 \pm 15.71$ & $75.00 \pm 8.51$ & $0.585^{\mathrm{a}}$ \\
\hline GC4 & $70.78 \pm 15.34$ & $70.29 \pm 15.98$ & $69.67 \pm 8.74$ & $0.621^{\mathrm{a}}$ \\
\hline GC5 & $66.65 \pm 17.51$ & $69.42 \pm 16.62$ & $78.07 \pm 5.73$ & $0.041^{\mathrm{a}}$ \\
\hline GC6 & $68.70 \pm 16.74$ & $71.71 \pm 14.76$ & $77.64 \pm 4.25$ & $0.183^{\mathrm{a}}$ \\
\hline GCavg & $69.48 \pm 16.41$ & $72.16 \pm 13.94$ & $76.07 \pm 5.30$ & $0.369^{\mathrm{a}}$ \\
\hline GCmin & $64.48 \pm 20.91$ & $65.89 \pm 20.82$ & $69.64 \pm 8.86$ & $0.727^{\mathrm{a}}$ \\
\hline
\end{tabular}

a ANOVA

GC1 - superonasal sector of the ganglion cell-inner plexiform layer, GC2 - superior sector of the ganglion cell-inner plexiform layer, GC3-superotemporal sector of the ganglion cell-inner plexiform layer, GC4 - inferotemporal sector of the ganglion cell-inner plexiform layer, GC5 - inferior sector of the ganglion cell-inner plexiform layer, GC6 - inferonasal sector of the ganglion cell-inner plexiform layer, GCavg-ganglion cell-inner plexiform layer average thickness, GCmin - ganglion cell-inner plexiform layer minimum thickness

Table III. Correlation with the stage of the disease $(N=46)$

\begin{tabular}{|lcc|}
\hline & Correlation coefficient $^{*}$ & $p$ \\
\hline GC1 & 0.152 & 0.192 \\
\hline GC2 & 0.098 & 0.403 \\
\hline GC3 & 0.129 & 0.269 \\
\hline GC4 & 0.098 & 0.401 \\
\hline GC5 & 0.203 & 0.081 \\
\hline GC6 & 0.209 & 0.071 \\
\hline GCavg & 0.112 & 0.340 \\
\hline GCmin & 0.058 & 0.619 \\
\hline
\end{tabular}

*Spearman's coefficient

$N$ - number of patients with Parkinson's disease, GC1 - superonasal sector of the ganglion cell-inner plexiform layer, GC2 - superior sector of the ganglion cell-inner plexiform layer, GC3 - superotemporal sector of the ganglion cell-inner plexiform layer, GC4 - inferotemporal sector of the ganglion cell-inner plexiform layer, GC5 - inferior sector of the ganglion cell-inner plexiform layer, GC6 - inferonasal sector of the ganglion cell-inner plexiform layer, GCavg-ganglion cell-inner plexiform layer average thickness, GCmin - ganglion cell-inner plexiform layer minimum thickness

tistically significant correlation between the stages of the disease and the thickness of GCL-IPL, as presented in Table III.

Correlation analysis between disease duration and the value of GC1, GC2, GC3, GC5, GC6, GCLavg, GCLmin was not statistically significant. However, there was a significant positive correlation between the duration of the PD and the thickness of the inferior (GC4) sector, as shown in Table IV.

\section{Discussion}

The most accessible dopaminergic neurons of the vertebrate CNS are the dopaminergic amacrine cells (DA cells) of the retina. Retinal dopamine has multi-
Table IV. Correlation with duration of the disease $(N=46)$

\begin{tabular}{|lcc|}
\hline & Correlation coefficient $^{*}$ & $p$ \\
\hline GC1 & 0.228 & 0.252 \\
\hline GC2 & 0.042 & 0.834 \\
\hline GC3 & 0.341 & 0.082 \\
\hline GC4 & 0.436 & 0.023 \\
\hline GC5 & 0.338 & 0.084 \\
\hline GC6 & 0.338 & 0.084 \\
\hline GCavg & 0.275 & 0.165 \\
\hline GCmin & 0.037 & 0.854 \\
\hline
\end{tabular}

*Spearman's coefficient

$N$ - number of patients with Parkinson's disease, GC1 - superonasal sector of the ganglion cell-inner plexiform layer, GC2 - superior sector of the ganglion cell-inner plexiform layer, GC3 - superotemporal sector of the ganglion cell-inner plexiform layer, GC4 - inferotemporal sector of the ganglion cell-inner plexiform layer, GC5 - inferior sector of the ganglion cell-inner plexiform layer, GC6 - inferonasal sector of the ganglion cell-inner plexiform layer, GCavg - ganglion cell-inner plexiform layer average thickness, GCmin - ganglion cell-inner plexiform layer minimum thickness

ple roles in vision $[4,22,27]$. Dopaminergic amacrine cells exhibit two classes of intrinsic bursting in the dark, shaped by inhibitory synaptic inputs, and two classes of light responses, ON-transient and ON-sustained, as well as light-independent activity, tuned to mediate specific dopaminergic functions in vision. The functional heterogeneity revealed in dopaminergic amacrine cells provides a cellular basis for the multiple roles of dopaminergic amacrine neurons in vision and is likely a general property of dopaminergic neurons throughout the CNS $[2,5,12,23]$.

Localization of amacrine cells is very important. Specifically, placed in the inner plexiform layer, the layer which, with the advent of the latest version of 
the OCT and segmentation of retinal layers, can be measured together with the ganglion cell layer $[8,19]$. This made amacrine cells even more accessible. OCT technology might prove useful in the diagnosis and monitoring changes in patients with PD by monitoring changes in the GCL-IPL layer.

Inzelberg et al. showed a reduction in the inferotemporal peripapillary retinal nerve fiber layer (RNFL) thickness and pointed out that changes in the level of internal layers of the retina can be of importance for the monitoring PD progression for the first time [17]. Some subsequent studies reported also evidence of peripapillary RNFL thinning in patients with PD $[5,21]$. RNFL thickness in patients with PD was also monitored by Lee et al. who proved the presence of statistically significant thinning of the RNFL and its association with duration and severity of disease. Lee was among the first ones who questioned whether retina could be a biomarker for disease progression [21]. Kaur et al. were among the first ones who in addition to RNFL also monitored the changes in the level of GCL-IPL concluding that GCL-IPL complex may be a more reliable parameter than RNFL thickness for structural alterations at the retina of patients with PD [18].

Currently there are not much data in the literature on the thickness of the GCL-IPL layer in PD. The loss of dopaminergic amacrine cells, i.e. failure of stimulation of metabotropic receptors with dopamine and subsequent failure in transition from scotopic to photopic vision by setting the gain of the retina in PD has been demonstrated [13]. The retinal thickness as a biomarker for PD has been investigated in some previous studies [26] and is currently under investigation in a clinical trial (https://clinicaltrials.gov/ct2/show/NCT02443779).

Our results indicate a statistically significant thinning of not only average and minimum GCL-IPL in PD, but also in all 6 sectors. Our results are similar to the results of Bayhan et al. who also pointed out thinning but unlike us they monitored ganglion cell complex on SD-OCT (RTVue-100), which includes the RNFL [7]. Cirrus SD-OCT device (model 4000 software version 6.0, Carl Zeiss Meditec, Inc.) used in our study can perform segmentation by subtracting RNFL from the complex, making the sole monitoring of GCL-IPL layer possible. We used ganglion cell analysis algorithm, which can automatically segment macular GCL-IPL thickness with excellent intervisit reproducibility $[7,19,25]$. Previous studies have evaluated GCL or IPL using different segmentation algorithms with similar results [10]. Sari et al. also indicated that the retinal dopaminergic neurodegeneration in patients with PD can be detected with GCL-IPL macular thickness measurements [28]. Our results are however not fully consistent with the results of Sari, who got a statistically significant correlation between the duration and severity of PD and the GCL-IPL thinning. Their conclusion was that GCL-IPL may be used to follow disease progression and the efficacy of neuroprotective treatment in patients with PD. Unlike their results, our study did not confirm the correlation between thickness of GCL-IPL, PD severity and duration. We found only a focal positive correlation between disease duration and the thinning of the GC4 sector.

There was no statistically significant difference in values for GC1, GC2, GC3, GC4, GC6, GCavg and $\mathrm{GCmin}$ between the stages of the disease. The only statistically significant differences registered were between stages I and II ( $p=0.046)$, and stages II and III ( $p=0.012)$ at sector GC5. These results lead to the thinking whether lower sectors were more sensitive to dopaminergic neurodegeneration, i.e. whether amacrine cells located at lower sectors are more prone to the progression of the disease. Additional studies are necessary to follow progression of PD using thickness of GCL-IPL complex.

Even in the earliest stages, PD is accompanied by changes in the internal layers of the macula in the form of decreased GCL-IPL layer thickness. This may indicate a possible retinal dopaminergic neurodegeneration. There is no correlation between duration or severity of PD with thinning of the GCL-IPL complex. The inferior sector is the only sector where a statistically significant difference was registered in thickness values between the different stages of the disease. To assess the significance of the findings in the context of their role as a surrogate biomarker for disease progression, further studies are required, which would include a larger number of patients in all stages of the disease.

\section{Disclosure}

Authors report no conflicts of interest.

\section{References}

1. Armstrong RA. Visual symptoms in Parkinson's disease. Parkinsons Dis 2011; 2011: 908306. 
2. Aaker GD, Myung JS, Ehrlich JR, Mohammed M, Henchcliffe C, Kiss S. Detection of retinal changes in Parkinson's disease with spectral-domain optical coherence tomography. Clin Ophthalmol 2010; 4: 1427-1432.

3. Adam CR, Shrier E, Ding Y, Glazman S, Bodis-Wollner I. Correlation of inner retinal thickness evaluated by spectral-domain optical coherence tomography and contrast sensitivity in Parkinson disease. J Neuroophthalmol 2013; 33: 137-142.

4. Almer Z, Klein KS, Marsh LGerstenhaber M, Repka MX. Ocular motor and sensory function in Parkinson's disease. Ophthalmology 2012; 119: 178-182.

5. Altintas O, Işeri P, Ozkan B, Caglar Y. Correlation between retinal morphological and functional findings and clinical severity in Parkinson's disease. Doc Ophthalmol 2008; 116: 137-146.

6. Archibald NK, Clarke MP, Mosimann UP, Burn DJ. The retina in Parkinson's disease. Brain 2009; 132 (Pt 5): 1128-1145.

7. Bayhan HA, Bayhan AS, Tanık N, Gürdal C. The association of spectral-domain optical coherence tomography determined ganglion cell complex parameters and disease severity in Parkinson's disease. Curr Eye Res 2014; 39: 1117-1122.

8. Francoz M, Fenolland JR, Giraud JM, El Chehab H, Sendon D, May F, Renard JP. Reproducibility of macular ganglion cell-inner plexiform layer thickness measurement with cirrus HD-OCT in normal, hypertensive and glaucomatous eyes. Br J Ophthalmol 2014; 98 : 322-328.

9. Frederick JM, Rayborn ME, Laties AM, Lam DM, Hollyfield JG. Dopaminergic neurons in the human retina. J Comp Neurol 1982; 210: 65-79.

10. Garcia-Martin E, Larrosa JM, Polo V, Satue M, Marques ML, Alarcia R, Seral M, Fuertes I, Otin S, Pablo LE. Distribution of retinal layer atrophy in patients with parkinson disease and association with disease severity and duration. Am J Ophthalmol 2014; 157: 470-478.

11. Goetz CG, Poewe W, Rascol O, Sampaio C, Stebbins GT, Counsell C, Giladi N, Holloway RG, Moore CG, Wenning GK, Yahr MD, Seidl L Movement disorder society task force report on the Hoehn and Yahr staging scale: status and recommendations. The movement disorder society task force on rating scales for Parkinson's disease. Mov Disord 2004; 19: 1020-1028.

12. Hajee ME, March WF, Lazzaro DR, Wolintz AH, Shrier EM, Glazman S, Bodis-Wollner IG. Inner retinal layer thinning in Parkinson disease. Arch Ophthalmol 2009; 127: 737-741.

13. Hirasawa H, Betensky RA, Raviola E. Corelease of dopamine and GABA by a retinal dopaminergic neuron. J Neurosci 2012; 32 13281-13291.

14. Hoehn M, Yahr M. Parkinsonism: onset, progression and mortality. Neurology 1967; 17: 427-442.

15. Hughes AJ, Daniel SE, Kilford L, Lees AJ. Accuracy of clinical diagnosis of idiopathic Parkinson's disease: a clinico-pathological study of 100 cases. J Neurol Neurosurg Psychiatry 1992; 55: 181-184.

16. Inzelberg R, Ramirez JA, Nisipeanu P, Ophir A. Retinal nerve fiber layer thinning in Parkinson disease. Vis Res 2004; 44: 2793-2797.

17. Jankovic J. Parkinson's disease: clinical features and diagnosis. J Neurol Neurosurg Psychiatry 2008; 79: 368-376.

18. Kaur M, Saxena R, Singh D, Behari M, Sharma P, Menon V. Correlation Between Structural and Functional Retinal Changes in Parkinson Disease. J Neuroophthalmol 2015; 35: 254-258.
19. Koh VT, Tham YC, Cheung CY, Whang WL, Baskaran M, Saw S, Wong T, Aung M. Determinants of Ganglion Cell-Inner Plexiform Layer Thickness Measured by High-Definition Optical Coherence Tomography. Invest Ophthalmol Vis Sci 2012; 53: 5853-5859.

20. Langheinrich T, Tebartz van Elst L, Lagreze WA, Bach M, Lücking $\mathrm{CH}$, Greenlee MW. Visual contrast response functions in Parkinson's disease: evidence from electroretinograms, visually evoked potentials and psychophysics. Clin Neurophysiol 2000; 111: 66-74.

21. Lee JY, Ahn J, Kim TW, Jeon BS. Optical coherence tomography in Parkinson's disease: is the retina a biomarker? J Parkinsons Dis 2014; 4: 197-204

22. Matsui H, Udaka F, Tamura A, Oda M, Kubori T, Nishinaka K, Kameyama M. Impaired visual acuity as a risk factor for visual hallucinations in Parkinson's disease. J Geriatr Psychiatry Neurol 2006; 19: 36-40.

23. Moschos MM, Tagaris G, Markopoulos I, Margetis I, Tsapakis S, Kanakis M, Koutsandrea C. Morphologic changes and functional retinal impairment in patients with Parkinson disease without visual loss. Eur J Ophthalmol 2011; 21: 24-29.

24. Movement disorder society task force on rating scales for Parkinson's disease. The Unified Parkinson's Disease Rating Scale (UPDRS): status and recommendations. Mov Disord 2003; 18 : 738-750.

25. Mwanza J-C, Oakley JD, Budenz DL, Chang RT, Knight OJ, Feuer WJ. Macular ganglion cell-inner plexiform layer: automated detection and thickness reproducibility with spectral domain-optical coherence tomography in glaucoma. Invest Ophthalmol Vis Sci 2011; 52: 8323-8329.

26. Normando EM, Davis BM, De Groef L, Nizari S, Turner LA, Ravindran N, Pahlitzsch M, Brenton J, Malaguarnera G, Guo L, Somavarapu S, Cordeiro MF. The retina as an early biomarker of neurodegeneration in a rotenone-induced model of Parkinson's disease: evidence for a neuroprotective effect of rosiglitazone in the eye and brain. Acta Neuropathol Commun 2016; 4: 86.

27. Onofrj M, Bonanni L, Albani G, Mauro A, Bulla D, Thomas A. Visual hallucinations in Parkinson's disease: clues to separate origins. J Neurol Sci 2006; 248: 143-150.

28. Sari ES, Koc R, Yazici A, Sahin G, Ermis SS. Ganglion Cell-Inner Plexiform Layer Thickness in patients with Parkinson Disease and association with disease severity and duration. J Neuroophthalmol 2015; 35: 117-121.

29. Sartucci F, Orlandi G, Lucetti C, Bonuccelli U, Murri L, Orsini C, Porciatti $V$. Changes in pattern electroretinograms to equiluminant red-green and blue-yellow gratings in patients with early Parkinson's disease. J Clin Neurophysiol 2003; 20: 375-381.

30. Schraermeyer U, Kopitz J, Peters S, Henke-Fahle S, BlitgenHeinecke P, Kokkinou D, Schwarz T, Bartz-Schmidt KU. Tyrosinase biosynthesis in adult mammalian retinal pigment epithelial cells. Exp Eye Res 2006; 83: 315-321.

31. Yu JG, Feng YF, Xiang Y, Huang JH, Savini G, Parisi V, Yang WJ, Fu XA. Retinal nerve fiber layer thickness changes in Parkinson disease: a meta-analysis. PLoS One 2014; 9: e85718. 